\title{
Breaking a habit: A further role of the phonological loop in action control
}

\author{
Erina Saeki • Alan D. Baddeley • Graham J. Hitch • \\ Satoru Saito
}

Published online: 18 April 2013

(C) Psychonomic Society, Inc. 2013

\begin{abstract}
Recent research has suggested that keeping track of a task goal in rapid task switching may depend on the phonological loop component of working memory. In this study, we investigated whether the phonological loop plays a similar role when a single switch extending over several trials is required after many trials on which one has performed a competing task. Participants were shown pairs of digits varying in numerical and physical size, and they were required to decide which digit was numerically or physically larger. An experimental cycle consisted of four blocks of 24 trials. In Experiment 1, participants in the task change groups performed the numerical-size judgment task during the first three blocks, and then changed to the physical-size judgment task in the fourth. Participants in the continuation groups performed only the physical-size judgment task throughout all four blocks. We found negative effects of articulatory suppression on the fourth block, but only in the task change groups. Experiment 2 was a replication, with the modification that both groups received identical instructions and practice. Experiment 3 was a further replication using numerical-size judgment as the target task. The results showed a pattern similar to that from Experiment 1, with negative effects of articulatory suppression found only in the task change group. The congruity of numerical and physical size had a reliable effect on
\end{abstract}

E. Saeki $\cdot$ S. Saito

Department of Cognitive Psychology in Education,

Kyoto University, Kyoto, Japan

A. D. Baddeley · G. J. Hitch

Department of Psychology, University of York, York, UK

E. Saeki $(\bowtie)$

Department of Cognitive Psychology in Education, Graduate School of Education, Kyoto University, Yoshida-Hommachi, Kyoto 606-8501, Japan

e-mail: saekie@educ.kyoto-u.ac.jp performance in all three experiments, but unlike the task change, it did not reliably interact with articulatory suppression. The results suggest that in addition to its well-established role in rapid task switching, the phonological loop also contributes to active goal maintenance in longer-term action control.

Keywords Action control $\cdot$ Phonological loop

The executive control of mental processes offers one of the most challenging problems in cognitive psychology. Such control plays a central role in virtually all theoretical approaches to working memory (Miyake \& Shah, 1999), and within the Baddeley and Hitch (1974) model, it was initially assigned to the central executive component. Such an assignment does not, of course, provide an explanation, and since that time, researchers have made extensive attempts to give an account of the executive processes that appear to perform this controlling function (Baddeley, 1996, 2007; Kane \& Engle, 2003; Oberauer, 2007, 2009; Shallice, 2002; Stuss \& Knight, 2002).

In recent years, however, it has become clear that the control of action may in certain circumstances extend beyond the central executive to the phonological loop, a temporary storage system specialized for the retention of speech-based material. Evidence for this was provided by a series of experiments in which Baddeley, Chincotta, and Adlam (2001) studied the capacity to switch rapidly between two divergent tasks, a process that they initially assumed depended crucially on the central executive. They used concurrent tasks that were assumed to disrupt only the phonological loop, or both the loop and the executive. In their study, participants performed addition and subtraction tasks alternately in an experimental block. Performance in such alternating blocks was compared with that from pure blocks in which participants engaged in either addition or 
subtraction throughout. Articulatory suppression did not have any specific effects on the pure blocks, but it grossly disrupted performance on the alternating blocks, an effect that was abolished in blocks in which each digit was accompanied by the relevant plus or minus sign. This basic finding has been replicated and further studied by Emerson and Miyake (2003), Saeki and Saito (2004a), and Saeki, Saito, and Kawaguchi (2006).

Subsequent work has suggested that articulatory suppression prevented retrieving and activating the appropriate task goal at the point of switching (Goschke, 2000; Miyake, Emerson, Padilla, \& Ahn, 2004). However, a series of studies employing sequential task-switching paradigms have established that the negative effects of articulatory suppression are found only in situations in which the stimuli are bivalent, and with the same physical stimulus potentially cueing two different responses in which control is endogenous and dependent on the participant maintaining the correct sequence of responses (e.g., Bryck \& Mayr, 2005; Saeki \& Saito, 2004b). These studies extended the earlier research, based on simple alternation, to the more complex alternating-runs paradigm, in which participants were required to switch tasks every second trial (i.e., task A, task A, task B, task B, ...). In this situation, task-switch and task-repeat trials occur within the same experimental block, resulting in a task conflict in both types of trial. Articulatory suppression affected repetition and switch trials equally, indicating that the contribution of the phonological loop is not confined to the specific control processes at the point of switching. The phonological loop appears to underpin the global task control processes that resolve the task conflict caused by bivalent stimuli when tasks are intermixed.

In the present study, we further examined the contribution of the phonological loop to executive control in a "long-term" task conflict situation in which an action directed toward a current task goal competed with an action driven by a welllearned response or habit. Task conflict of this type is encountered in everyday life and is potentially different from the conflict studied in typical task-switching paradigms, which require participants to switch frequently back and forth between two broadly equivalent tasks. As Monsell (1996) pointed out, without goal-directed executive control, our thought and action would be "a prisoner of habit." The primary purpose of the present study was to examine the role of the phonological loop in avoiding such imprisonment.

For this purpose, we experimentally created a microhabit that favored one task over another when two tasks were based on the same set of stimuli. We began by having all participants practice a specified judgment regarding pairs of digits that differed in both physical and numerical size. Phase 1 of the experiment involved extensive practice on one of these judgments, followed by Phase 2, in which half of the participants continued with the same judgment, and the remainder switched to the alternative judgment. For each participant, the initial habit induction in Phase 1 was repeated across three blocks of trials. The crucial final block (i.e., Phase 2) was performed under baseline control conditions, under articulatory suppression, or under a nonarticulatory concurrent task (repeated foot tapping). This paradigm is analogous to the Baddeley et al. (2001) task-switching procedure, except that a task of rapid and frequent switching is replaced by a single switch extending over several trials. Considering that earlier work had shown no effect of articulatory suppression during a pure block in which participants performed one of two tasks throughout the block (Baddeley et al., 2001; Emerson \& Miyake, 2003; Saeki \& Saito, 2004a), one might hardly expect to find a disruptive effect of articulatory suppression from repeating the same task over many trials in the present study. In a pure-task block, participants could identify the task in all trials with quite high confidence (Rubin \& Meiran, 2005). Because they performed the same task as they had on the previous trial, the target task was always obvious on each trial in the pure-task block, even though the stimuli were bivalent. This low task uncertainty could result in less demand for task control in this situation. In our microhabit procedure described above, similarly, the task was always obvious on every trial. However, in the switch condition, a recently built-up habit had to be resisted in order to perform the new task judgment efficiently in the target block (Phase 2). The habit acquired before performing the target block could potentially lead to increased demand for task control, even when the same new task was performed over many trials. If this were the case, the phonological loop could influence the long-term task conflict condition, even though there was no need to maintain sequence order.

A previous study by Saeki (2007) provided general support for this proposal. In that study, participants were presented with a pair of digits on each trial. The digits differed in both numerical size and font size (e.g., 3 7), and participants were required to indicate which one was numerically or physically larger. Two groups were tested: a continuation group, who engaged in the numerical-size judgment task in both Phase 1 (the first three blocks), and Phase 2 (a fourth block), and a change group, who performed the physical-size judgment task in Phase 1 and then switched to the numerical-size judgment task in Phase 2. The study focused on performance in Phase 2. Saeki found that articulatory suppression had a negative effect in the task change group but not in the continuation group. However, although the stimuli for the numerical-/physicalsize tasks were bivalent, the balance was inevitably imperfect. For example, the numerical task takes slightly longer and involves more variable comparisons than the physical-size task, since the numerical distance between pairs of digits is more variable than the difference in their physical sizes. Previous research on short-term switching has shown 
asymmetries that depend on whether the switch is from an easier to a more difficult task, or vice versa (Schneider \& Anderson, 2010). Bearing these asymmetries in mind, it was important to ensure that the results of Saeki were due to change per se and were not an artifact of switching from an easier to a more difficult task. In Experiment 1, we achieved this by reversing the conditions used by Saeki, and consequently focusing in the crucial switch block on physical rather than numerical judgments. We addressed this issue in Experiment 1.

In addition to the above-mentioned issue, we considered a further potentially complicating aspect of the design of Saeki (2007). In that study, participants were informed about the task requirements over the whole of the forthcoming cycle. This involved instructions for both judgment tasks in the change condition, but only for one set of instructions in the continuation group. Therefore, it might be that, irrespective of a microhabit that was built up during Phase 1, participants in the task change group might have suffered from task conflict caused by receiving two sets of task instructions, as compared with one set in the continuation group, in which task conflict was absent. It is already known that giving instructions for two tasks rather than one requires participants to construct a more complex task model, resulting in poor performance even when they are required to perform only one task (Duncan et al., 2008). We addressed this concern in Experiments 2 and 3 by replicating Experiment 1 and Saeki's study, respectively, but giving the same initial instructions and practice to both groups.

Another separate issue concerns the size congruity effect in the size judgment task. Previous studies have consistently reported that reaction times are longer in incongruent trials (e.g., a numerically larger digit is presented in a smaller font) than in congruent trials, regardless of the type of judgment (e.g., Besner \& Coltheart, 1979; Dehaene \& Akhavein, 1995; Henik \& Tzelgov, 1982; Tzelgov, Meyer, \& Henik, 1992). This suggests that interference can occur on the basis of activation triggered by the irrelevant dimension. The source of this conflict effect does, of course, differ from the main focus of our study, as it is a Stroop-like effect that changes unpredictably on every trial — a conflict effect that presumably reflects the inherent microstructure of the task. This contrasts with long-term conflict between two consistent and explicit task instructions, which is the principal focus of our study. As such, it is unclear to what extent it might be sensitive to our concurrent tasks.

Saeki (2007) found that the effects of articulatory suppression did not interact with size congruity, suggesting that the phonological loop does not contribute to resolving interference arising from activation driven by the irrelevant dimension. However, it would clearly be unwise to draw strong conclusions from a null result, and in the experiments that follow, we further explored the question of a possible interaction between articulatory suppression and size congruity.

To foreshadow the results, we found that articulatory suppression had negative effects in the change group, in which the switch was from a numerical to a physical judgment (Exp. 1), and we certified that this effect was not caused by the more complex instructions given to the change group (Exps. 2 and 3). With respect to an interaction between size congruity and articulatory suppression, our results suggest that articulatory suppression had little impact on the size congruency effect. We will discuss these results as suggesting that the phonological loop contributes to goal maintenance in longer-term action control.

\section{Experiment 1}

In this study, we examined the effects of articulatory suppression on the physical judgment task following a long run of trials on the numerical judgement task. Participants were required to successively perform four blocks in each cycle of the experiment (see Fig. 1). Both groups were given instructions regarding the whole four-block cycle. Hence, those in the continuation group were instructed to perform only the physical-size judgment task, whereas participants in the change group were instructed regarding both the numerical- and physical-size judgment tasks. The latter group performed the numerical-size task during the first three blocks (Phase 1) and switched to the physical-size judgment task in the final, fourth block (Phase 2). The critical comparison between groups concerned the fourth block. Performance in this block was tested under three concurrent-task conditions: control (no secondary task), articulatory suppression, and foot tapping. The foot-tapping condition was included in order to investigate the effects on performance of a task that resembled articulatory suppression, in requiring a repetitive activity that might be expected to place a modest additional demand on executive resources while avoiding a direct verbal component. If suppression were to differentially disrupt performance in the switch group, this would suggest a role for the phonological loop in long-term goal maintenance extending beyond that already shown in rapid-switching tasks.

\section{Method}

Participants The participants were 36 undergraduate students from the University of York. Half were assigned to the continuation group and the other half to the change group.

Apparatus The experiment was conducted with E-Prime 2.0 software (Psychology Software Tools, Inc., Sharpsburg, PA) 


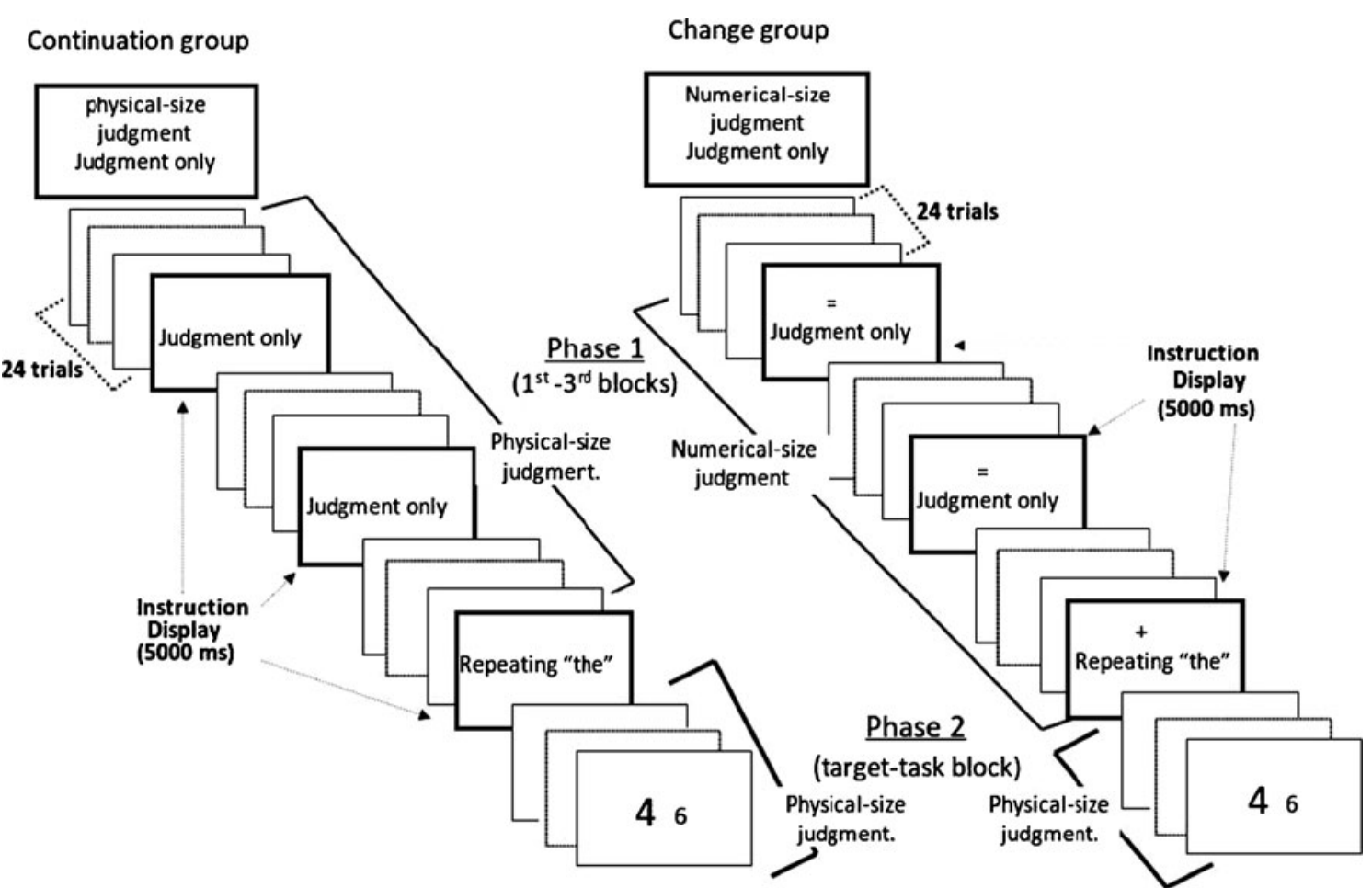

Fig. 1 Schematic illustration of the sequences of task blocks used in the continuation group (left) and the change group (right) in Experiment 1. An instruction display was inserted between blocks. The instruction display in the continuation group consisted of a simple sentence detailing the concurrent-task condition (judgment only, repeating "the," or foot tapping). The instruction display in the change group consisted of a sentence detailing the concurrent-task conditions

running on a computer that displayed stimuli on monitors and registered responses from a serial response box.

Tasks and stimuli The stimuli consisted of pairs of digits from 2 to 8 , excluding 5 , that differed in both physical and numerical size (e.g., 7 3, 6 2). The pairs were presented in white on a black background. In sizecongruent trials, the numerically larger digit was also the physically larger one, whereas in size-incongruent trials, the numerically larger digit was the physically smaller one, or vice versa. The physically larger digits were $13 \mathrm{~mm}$ wide and $18 \mathrm{~mm}$ high, and the smaller digits were $8 \mathrm{~mm}$ wide and $12 \mathrm{~mm}$ high. Participants were required to decide which digit in each pair was numerically larger in the numerical-size judgment task, and to decide which digit in each pair was physically larger in the physical-size judgment task. They responded by pressing the extreme left or right key on the response box with their right or left index finger to indicate the target digits

Procedure The sequence of events in a cycle is shown in Fig. 1 and is based on that used by Saeki (2007). Each cycle comprised four blocks of 24 trials, containing 12 congruent and a sign $(=$ or +$)$ pertaining to the task. The equal sign indicated that participants were to perform the same task in the next block as in the previous block, whereas the plus sign indicated that they had to change to the other task in the next block. Changes in the concurrent-task condition (and of the task, in the change group) were required only in the fourth block. Only performance in the fourth block was analyzed

and 12 incongruent pairs in an unpredictable order. For the continuation group, the initial instructional display was presented for $5,000 \mathrm{~ms}$ and indicated that only the physical-size judgment task was required. This was followed after 24 trials by a second 5,000-ms instructional display, again indicating a physical judgment, and after a further 24 trials by a third identical display. The final block was preceded by an instructional display that cued the concurrent task to be performed at the same time as making the physical-size judgments (i.e., suppression, foot tapping, or control). Participants were required to start the corresponding dual task immediately if the instructional display indicated suppression or foot tapping. A fixation point was presented at the center of the screen for 1,000 ms between the trials and between the instructional display and the first trial in each block. The change group's procedure was exactly the same, except that participants were told about both judgment tasks before being given the initial instruction to judge numerical size. The subsequent instructional displays were equivalent to those in the continuation group, except that they also contained a cue instructing participants either to continue with the same type of judgment $(=)$ or, just prior to the fourth block, to change to the alternative judgment $(+)$. Participants were not told in advance that this 
change would only occur in the final block, during which they also performed one of the concurrent task conditions at the same time as making physical-size judgments.

Participants performed two cycles under each concurrenttask condition, making six in all. In the control condition, participants performed the physical-size judgment task without any dual-task requirement. Under articulatory suppression, they vocalized "the" repeatedly, approximately twice per second, while doing the judgment task. In the tapping condition, they tapped with their foot at the same pace as in the suppression conditions. Three participants in each group were assigned to each of the six permutations of concurrenttask order.

At the beginning of the experiment, participants practiced each secondary task in time to a metronome until they were able to perform the task correctly. This was followed by a short practice to ensure that they had understood the instruction display. The participants in the continuation group performed 12 practice trials in each of the dual-task conditions following the instruction display (the order was control, articulation, and tapping). The participants in the change groups practiced the numerical-size judgment task first and then the physical-size judgment task, each in the control condition; participants then performed the numerical-size judgment task with articulatory suppression, and finally the physical-size judgment task with tapping, with appropriate instruction displays between blocks. Participants were required to start the dual task as soon as cued by the display and before the presentation of the first stimulus in the next block. They were instructed to respond to each stimulus as rapidly as possible while avoiding errors.

\section{Results}

Only the results of Phase 2 (a total of 48 trials under each concurrent-task condition) were analyzed here. ${ }^{1}$ As Table 1 shows, error rates were generally low in all conditions (less than about $5 \%$ ) and did not contradict the results for reaction times (RTs); that is, we found no speed-accuracy trade-offs (see Table 1). Twelve participants in the continuation group and two participants in the change group performed the tasks without making a mistake. Therefore, we report only the analyses of RTs. For the first trial in each block, incorrect responses and responses immediately following an error were excluded

\footnotetext{
${ }^{1}$ For completeness' sake, we also analyzed the reaction time results for Phase 1. Important points to bear in mind are that concurrent tasks were not manipulated in Phase 1 and that all participants performed the task under the silent control condition through Phase 1 in all experiments. The main purpose of the analysis was to show that the effect of size congruity was obtained in Phase 1 (see the Table 4).
}

from the analyses. Any RT more than three standard deviations from the individual mean for each block in each condition was also excluded (4.2\% of otherwise correct trials). The effect size measure reported is partial eta-squared $\left(\eta_{\mathrm{p}}{ }^{2}\right)$.

Figure 2 shows mean RTs as a function of group (continuation and change), size congruity (congruent and incongruent), and concurrent task condition (control, articulatory suppression, and foot tapping). The RT data were submitted to a three-way mixed analysis of variance (ANOVA) with Group as a between-subjects factor. The ANOVA yielded a main effect of group, $F(1,34)=6.43$, mean squared error $(M S E)=31,293.48, p<.05, \eta_{\mathrm{p}}{ }^{2}=.16$, with longer RTs in the change group; a main effect of size congruity, $F(1,34)=$ $77.14, M S E=1,081.34, p<.001, \eta_{\mathrm{p}}{ }^{2}=.69$, with longer RTs on incongruent trials; and a main effect of condition, $F(2$, $68)=16.70, M S E=3,316.59, p<.001, \eta_{\mathrm{p}}{ }^{2}=.33$, with RTs being slowest under suppression and fastest in the control condition.

The effect of concurrent-task conditions appeared to be restricted to the change group. Thus, we found a significant interaction between concurrent task and group, $F(2,68)=$ 16.45 MSE $=3,316.59, p<.001, \eta_{\mathrm{p}}{ }^{2}=.33$, and an analysis of simple effects revealed a significant effect of concurrenttask condition for the change group, $F(2,34)=24.30, M S E$ $=4,563.16, p<.001, \eta_{\mathrm{p}}{ }^{2}=.59$, but not for the continuation group, $F<1$. To separate out the specific effects of articulatory suppression from general dual-task demands, we conducted two $2 \times 2$ mixed ANOVAs with the factors Group (continuation and change) and Concurrent Task (control and tapping or articulatory suppression and tapping). The comparison between control and tapping showed a significant interaction, $F(1,34)=12.87, M S E=2,426, p=$ $.001, \eta_{\mathrm{p}}{ }^{2}=.27$, indicating that tapping slowed RTs relative to the control condition in the change group. The contrast between articulatory suppression and tapping also yielded a significant interaction, $F(1,34)=$ 7.94, $M S E=1,482, p<.01, \eta_{\mathrm{p}}{ }^{2}=.19$, indicating that articulatory suppression had a more disruptive effect than tapping in the change group.

Size congruity interacted with group, $F(2,34)=18.75$, $M S E=1,081.34, p<.001, \eta_{\mathrm{p}}{ }^{2}=.36$, in that the congruity effect was larger for the change group. No other significant interactions emerged, $F \mathrm{~s}<1$.

Finally, to check whether the disruptive effect of articulatory suppression in the change group persisted throughout Phase 2, we divided RTs into the first and second halves of the final block (see Table 2 ) and conducted a $3 \times 2$ ANOVA with the factors Concurrent Task and Trial Position (first and second). The interaction did not reach significance, $F<1$, indicating that the negative effects of articulatory suppression did not differ between the first and second halves of Phase 2. 
Table 1 Mean error rates for the different tasks in each condition of Experiments 1-3

\begin{tabular}{|c|c|c|c|c|}
\hline \multirow[b]{2}{*}{ Condition } & \multicolumn{2}{|c|}{ Task Continuation Group } & \multicolumn{2}{|c|}{ Task Change Group } \\
\hline & Congruent & Incongruent & Congruent & Incongruent \\
\hline \multicolumn{5}{|l|}{ Experiment 1} \\
\hline Control & 0 & .004 & 0 & .025 \\
\hline Articulation & .002 & .005 & 0 & .030 \\
\hline Tapping & .002 & .005 & .004 & .023 \\
\hline \multicolumn{5}{|l|}{ Experiment 2} \\
\hline Control & .002 & .016 & .002 & .019 \\
\hline Articulation & .002 & .007 & .012 & .030 \\
\hline Tapping & .014 & .012 & .016 & .005 \\
\hline \multicolumn{5}{|l|}{ Experiment 3} \\
\hline Control & .007 & .051 & .016 & .037 \\
\hline Articulation & .012 & .025 & .025 & .028 \\
\hline Tapping & .019 & .046 & .012 & .051 \\
\hline
\end{tabular}

\section{Discussion}

The results of this experiment replicated the main findings of Saeki (2007) using physical-size judgment rather than numerical-size judgment in the final block of trials. Thus, neither articulatory suppression nor tapping had any effect in the continuation group, in which participants repeated the same judgment task during all four blocks. On the other hand, both concurrent tasks increased RTs in the change group, in which participants changed task in the fourth block, with the effect being significantly bigger for suppression than for tapping. By replicating this aspect of Saeki's results, using the same tasks but with the task change in the opposite direction, we could rule out the possibility that the original findings were due to the change group switching from an easier to a more difficult task. We assume that during the first three blocks the participants built up a microhabit for the competing task, and consequently, when they were required to perform the target task in Phase 2, the task goal from Phase 1 was prone to intrude in Phase 2, increasing goal conflict. The effect of articulatory suppression suggests that the phonological loop plays an important role in protecting the current task goal from a competing and irrelevant task goal, over and above a smaller effect from the concurrent tapping task.

Turning to the effects of size congruity, these showed a somewhat different pattern from that uncovered by Saeki (2007): RTs were longer in incongruent trials, as before, but unlike in Saeki's study, the congruity effect was significantly bigger for the change group. However, it is noteworthy that, as for Saeki, articulatory suppression did not interact with size congruity, implying that the control processes supported by the phonological loop are different from the processes that resolve response ambiguity in incongruent trials.
Fig. 2 Mean reaction times for the continuation group (left) and the change group (right) in the control, articulatory suppression, and tapping conditions of Experiment 1. The bars represent standard errors

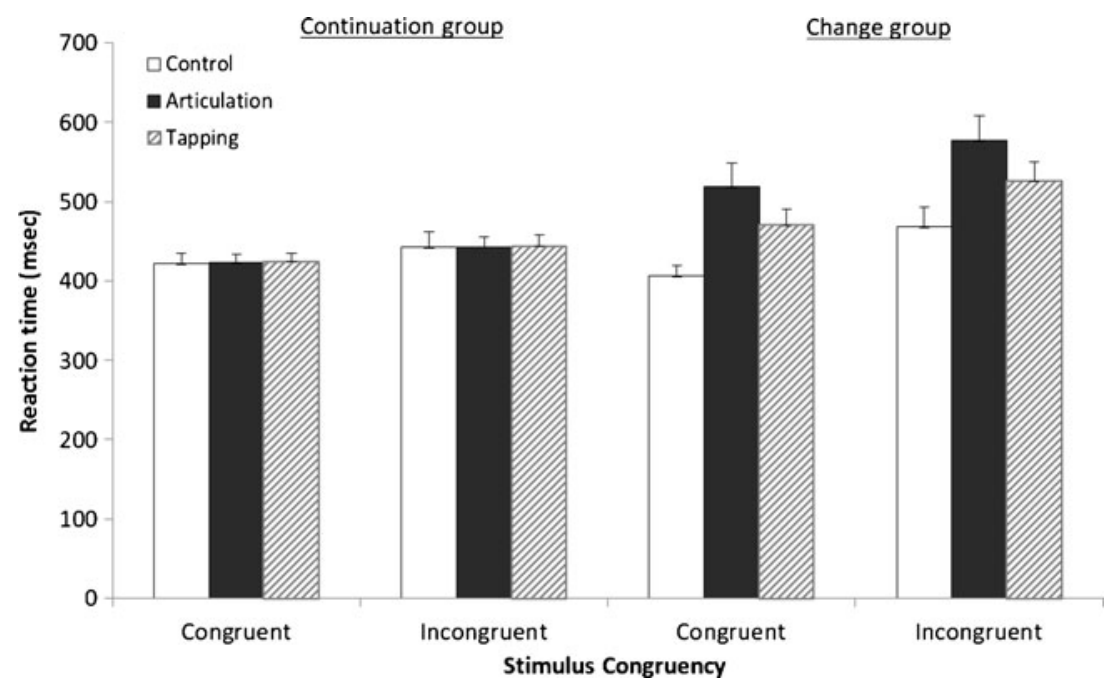


Table 2 Mean reaction times in the control, articulatory suppression, and tapping conditions in the change groups across Experiments 1-3, presented separately for the first and the second half of Phase 2

\begin{tabular}{|c|c|c|c|c|c|c|}
\hline & \multicolumn{2}{|l|}{ Control } & \multicolumn{2}{|c|}{ Articulatory Suppression } & \multicolumn{2}{|l|}{ Tapping } \\
\hline & First & Second & First & Second & First & Second \\
\hline Experiment 1 & 439.38 (18.17) & $434.74(18.06)$ & $555.24(29.31)$ & $540.46(34.10)$ & $496.54(21.00)$ & $500.30(22.24)$ \\
\hline Experiment 2 & $431.63(19.28)$ & $409.92(20.48)$ & $585.21(46.92)$ & $512.31(32.43)$ & $492.91(27.32)$ & $454.11(21.21)$ \\
\hline Experiment 3 & $542.39(24.01)$ & $521.73(20.24)$ & $607.11(27.13)$ & $599.39(27.77)$ & $555.80(21.78)$ & $538.14(19.20)$ \\
\hline
\end{tabular}

Standard errors are in parentheses.

\section{Experiment 2}

In this experiment, we again examined the effects of articulatory suppression on performance of the physical-size judgment task in Phase 2. However, unlike in Experiment 1, all participants were given exactly the same instructions and practice before starting the experimental blocks. Thus, regardless of task group, the meaning of the plus (switch strategy) and equal (continue) signs were explained, and instruction displays that included the plus or the equal sign were inserted appropriately between blocks during the practice trials and during the test trials. If the complexity of the instructions were responsible for the increased task control effects in Experiment 1, the negative effects of articulatory suppression should be observed in both the continuous and change groups in this experiment.

\section{Method}

The method and procedure were identical to those of Experiment 1, except that participants in both groups were given the same instructions and practice as the change-task group in Experiment 1. Thus, the practice phase consisted of four blocks of 12 trials. Participants started with the numerical judgment task under single-task conditions, followed by the physical judgment task, also under single-task conditions. In the third practice block, they performed the numerical-size judgment task under articulatory suppression, and in the final practice block, they did the physicalsize judgment task with tapping. An appropriate instruction display was presented at the start of each block. In the experiment proper, the change instructions never appeared to participants in the continuation group, and consequently they performed the physical-size judgment task throughout. However, they were not forewarned that they would never have to perform the alternative task.

The participants were 36 students from the University of York who were assigned to two groups: continuation $(N=18)$ and change $(N=18)$. The participants in the continuation group were required to perform only the physical-size judgment task, whereas those in the change groups were required to change from the numerical-size judgment task to the physical-size judgment task in Phase 2.

\section{Results}

Accuracy was again very high (see Table 1), and seven participants in the continuation group and seven in the change group performed the tasks without making a mistake. The RT results were analyzed in the same manner as in Experiment 1, and $6.2 \%$ of the responses were discarded from the analysis as outliers, using the same criterion as before. Figure 3 shows mean RTs as a function of group, size congruity, and concurrent-task condition (see also Table 2). An ANOVA revealed significant main effects of concurrent task, $F(2,68)=24.0, M S E=6,075, p<.001, \eta_{\mathrm{p}}{ }^{2}$ $=.14$, and size congruity, $F(1,34)=90.4, M S E=535, p<$ $.001, \eta_{\mathrm{p}}{ }^{2}=.73$, as in Experiment 1 . However, although RTs tended to be slower for the change group, the effect of change was not significant, $F(1,34)=1.95, M S E=$ $40,101, p=.17, \eta_{\mathrm{p}}{ }^{2}=.05$. The three-way interaction was also not significant, $F(2,68)=1.43, M S E=450, p=.25, \eta_{\mathrm{p}}{ }^{2}$ $=.04$, as in Experiment 1, allowing us to focus on the twoway interactions.

Once again, we found a significant interaction between concurrent task and group, $F(2,68)=5.79, M S E=6,075$, $p<.01, \eta_{\mathrm{p}}{ }^{2}=.15$. An analysis of simple effects revealed significant effects of concurrent-task condition in both the change group, $F(2,34)=15.8, M S E=9,472, p<.001, \eta_{\mathrm{p}}{ }^{2}=$ .48 , and the continuation group, $F(2,34)=11.5, M S E=$ $2,678, p<.001, \eta_{\mathrm{p}}{ }^{2}=.40$. Further $2 \times 2$ analyses pointed to the source of the interaction as being the contrast between suppression and tapping. Thus, when tapping was contrasted with control, a significant dual-task effect was apparent, $F(1,34)=24.9, M S E=1,946, p<.001, \eta_{\mathrm{p}}{ }^{2}=.42$, but no interaction with group, $F(1,34)<1$. However, when suppression was contrasted with tapping, we found a significant main effect of interfering task, $F(1,34)=8.82, M S E=$ $2,885, p<.01, \eta_{\mathrm{p}}{ }^{2}=.20$, as well as a significant interaction with group, $F(1,34)=8.83, M S E=2,885, p<.01, \eta_{\mathrm{p}}{ }^{2}=.20$. Suppression led to significantly longer RTs than did tapping in the change group, $t(17)=3.25, p<.01$, but not in the 
Fig. 3 Mean reaction times for the continuation group (left) and the change group (right) in the control, articulatory suppression, and tapping conditions of Experiment 2. The bars represent standard errors

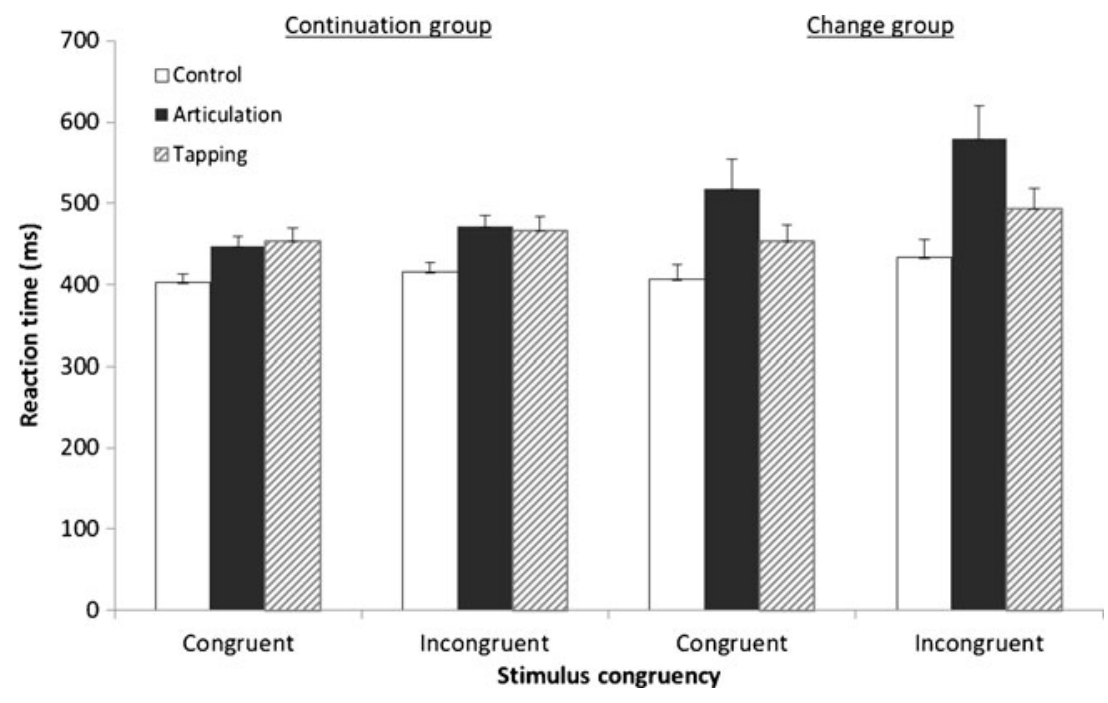

continuation group, $t(17)<1$. The contribution of suppression to the interaction with group replicates the main finding of Experiment 1 . However, whereas tapping previously had also made a contribution to the interaction, it did not do so here.

As in Experiment 1, a significant interaction emerged between group and size congruity, $F(1,34)=18.0, M S E$ $=535, p<.001, \eta_{\mathrm{p}}{ }^{2}=.35$. Once again, the congruity effect was significant in each group and was larger in the change group. Thus far, the results broadly replicated Experiment 1. However, one notable exception was the presence here of a significant interaction between size congruity and concurrent-task condition, $F(2,68)=$ 5.71, $M S E=450, p<.01, \eta_{\mathrm{p}}{ }^{2}=.14$. Further analysis showed that the congruity effect was significant in all three concurrent-task conditions, with the interaction reflecting a larger congruity effect under articulatory suppression.

Finally, as in Experiment 1, we conducted a $3 \times 2$ ANOVA with the factors Concurrent-Task Condition and Within-Block Position (first half and second half) to look in more detail at the effects of articulatory suppression in the change group (see Table 2). In contrast to the previous findings, the interaction was significant, $F(2,34)=3.83$, $M S E=1,849, p<.05, \eta_{\mathrm{p}}{ }^{2}=.18$. Post hoc analyses showed that articulatory suppression led to the longest RTs in both halves, but that RTs under articulatory suppression were faster in the second half.

\section{Discussion}

In this experiment, we used the same instructions and practice blocks for both the change group and the continuation group. The main features of the results were the same, but with some interesting exceptions. Thus, consistent with Saeki (2007) and with Experiment 1, suppression had a bigger disruptive effect on performance in the change group. This finding is further support for the hypothesis that the phonological loop plays a role in maintaining the task goal in the face of interference from a competing goal. One slight difference in outcomes was the emergence of some evidence that the effect of suppression on the change group declined over time in the present experiment. We will defer discussion of this observation until after establishing its reliability through further experimentation.

A further similarity in outcomes was the observation of a larger effect of size congruity in the change group. However, unlike in Experiment 1, the congruity effect interacted with concurrent task here, being somewhat greater under articulatory suppression. We will also address this discrepancy in the General Discussion.

\section{Experiment 3}

Our final experiment was motivated by the need to ensure that our results, based on switching from a numerical to a physical task, could also be found when the switch was in the opposite direction. The two groups in Saeki (2007) received different instructions prior to the experiment, as ours had in Experiment 1. Thus, Experiment 3 was identical to Experiment 2, in which all participants were given exactly the same instructions, except that participants in the continuation group performed the numerical-size judgment task throughout, and participants in the change group performed the physical-size judgment task for the first three blocks in each cycle, switching to the numerical task in the final block. Once again, our principal focus was on whether articulatory suppression would have a greater effect on the change group in the final block. 


\section{Method}

The method and procedure were identical to those used in Experiment 2, except that the physical-size and numericalsize judgment tasks were reversed. The participants comprised 36 students from the University of York, assigned to two groups: continuation $(N=18)$ and change $(N=18)$.

Results

Error rates were once again low (see Table 1), and one participant in the continuation group and one in the change group performed the tasks without making a mistake. Our analysis was confined to the RT data (see Table 2 and Fig. 4). In all, $9 \%$ of the responses were discarded from the RT analysis as outliers. As in Experiments 1 and 2, the three-way ANOVA revealed significant main effects of concurrent task, $F(2,68)=$ 13.4, MSE $=3,355, p<.001, \eta_{\mathrm{p}}{ }^{2}=.28$, and size congruity, $F(1,34)=76.7, M S E=1,146, p<.001$, $\eta_{\mathrm{p}}{ }^{2}=.69$. No main effect of group was apparent, $F(1$, $34)=2.16, M S E=39,383, p=.15, \eta_{\mathrm{p}}{ }^{2}=.05$, but a significant interaction again was found between concurrent-task condition and group, $F(2,68)=2.98$, $M S E=3,355, p<.05, \eta_{\mathrm{p}}{ }^{2}=.08$, with only the change group suffering significant disruption from articulatory suppression. Further analysis revealed a significant main effect of concurrent-task condition in the change group, $F(2,34)=30.9, M S E=1,578, p<.001, \eta_{\mathrm{p}}{ }^{2}=.64$, and no corresponding effect in the continuation group, $F(2$, $34)=1.23, M S E=5,132, p=.30, \eta_{\mathrm{p}}{ }^{2}=.06$. As before, the interaction was explored using $2 \times 2$ (Group $\times$ Condition) analyses. When tapping was contrasted with control, we found no significant effect of concurrent task, $F(1,34)<1$, and no interaction with group, $F(1$,
$34)<1$. However, when suppression was contrasted with tapping, suppression led to significantly more interference than did tapping, $F(1,34)=14.53, M S E=$ $3,718, p<.001, \eta_{\mathrm{p}}{ }^{2}=.30$, and this difference was marginally greater in the change group, $F(1,34)=$ $2.99, M S E=3,718, p=.09, \eta_{\mathrm{p}}^{2}=.08$. Pairwise comparisons showed that RTs were significantly longer with suppression than with tapping in the change group, $t(17)$ $=5.24, p<.001$, but no significant difference was observed in the continuation group, $t(17)=1.21, p=$ .24. The tendency for suppression to have a greater interfering effect on the change group was slightly less clear-cut than before, but essentially it replicated the earlier findings. As in Experiment 2, but not Experiment 1 , we found no evidence that tapping had a greater effect on the change group.

Unlike in Experiments 1 and 2, no significant interaction between size congruity and group emerged, $F(1,34)<1$. Size congruity did, however, interact with concurrent-task condition, $F(2,68)=6.44, M S E=589, p<.01, \eta_{\mathrm{p}}{ }^{2}=.15$, and we also found also a significant three-way interaction, $F(2,68)=4.33, M S E=589, p<.05, \eta_{\mathrm{p}}{ }^{2}=.11$. However, further analyses revealed that these interaction effects were quite different from any of the previous results. Thus, the two-way interaction reflected a larger congruity effect in the control condition, and the three-way interaction reflected a tendency for the congruity effect in the tapping condition to be bigger in the change group (see Fig. 4).

Finally, RTs for the change group were examined further to see whether the effect of articulatory suppression persisted throughout the whole block of trials (see Table 2). A $3 \times 2$ ANOVA with the factors Concurrent-Task Condition and Within-Block Position (first half and second half) showed that the interaction was not significant, $F(2,34)<1$, indicating that the disruptive effect did indeed persist.
Fig. 4 Mean reaction times for the continuation group (left) and the change group (right) in the control, articulatory suppression, and tapping conditions of Experiment 3. The bars represent standard errors

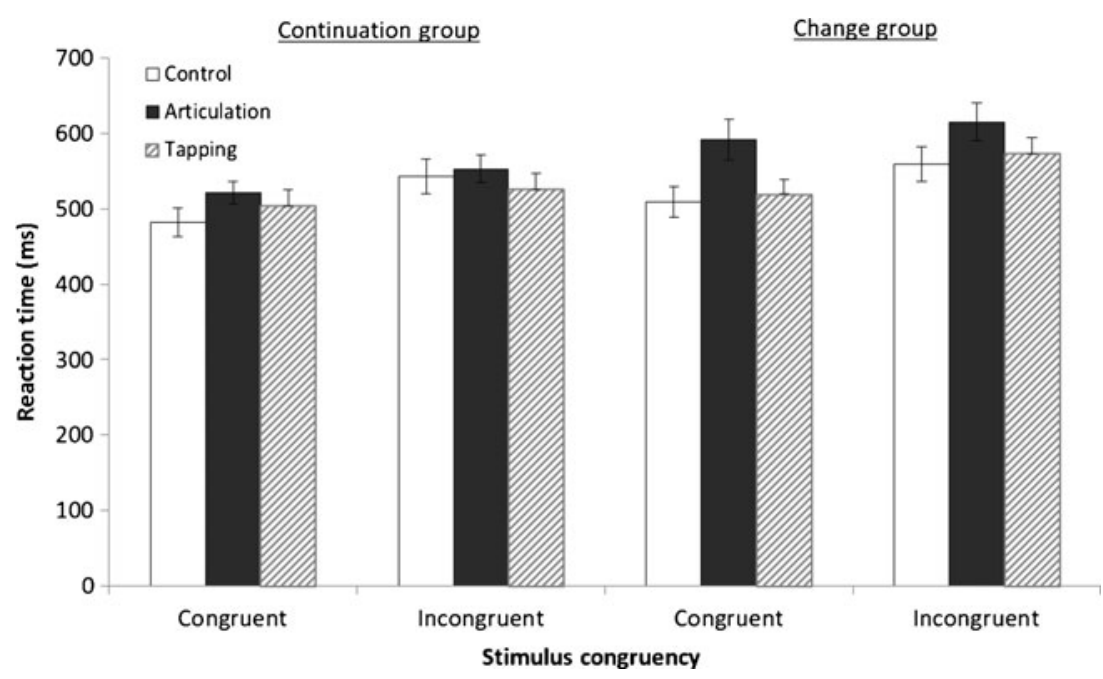




\section{Discussion}

These results help clarify the overall empirical picture. Most importantly, they provide further evidence of an effect of articulatory suppression that is restricted to participants who performed a competing judgment task for some time previous to a task change. The results also provide further evidence that the effect of suppression is long-lasting, persisting over trials involving the competing task.

Turning to the effects of size congruity, we saw a different picture, though one that was slightly less clear. The effect of congruity itself was highly robust, consistent with the previous data. However, the interactions involving congruity presented a more complex picture. First, we note that no two-way interaction took place between size congruity and group, unlike the interactions in Experiments 1 and 2, which showed a larger congruity effect in the change group. Second, although we did observe an interaction between congruity and concurrent task, this reflected an enhanced effect in the tapping condition, in contrast to the enhanced effect with suppression found in Experiment 2.

\section{General discussion}

We begin by summarizing the results of our three experiments and combining them with the earlier study by Saeki (2007), in order to establish the consistency across experiments of answers to the issues raised in the introduction. A summary of the results of the main three-way ANOVA on RTs is shown in Table 3. As can be seen, the two most consistent findings are the interaction between group and concurrent task (which reflected a greater effect of articulatory suppression in change groups), and the congruity effect (reflecting longer RTs for incongruent stimuli). Another notable result is the interaction between group and size congruity, which was significant only when the target task was the physical-size judgment in Experiments 1 and 2 (reflecting a larger congruity effect in change groups).
The role of the phonological loop in action control

The central question of our study concerns the role of the phonological loop in the control of action, focusing on its capacity to resist interference from an earlier habit. The results in all three experiments and in Saeki (2007) are consistent with the assumption that suppression operates by disrupting a mechanism that may be used to protect responding from disruption from prior habits. Suppression has no reliable effect when the mapping of responses is based on a continuation of a recently acquired habit, but it disrupts performance following a switch to a new mapping, showing the predicted interaction between suppression and task switching. One could argue that the novelty of the task in Phase 2 increased its difficulty, and that this increase in difficulty led to the effect of articulatory suppression in the change groups. However, this alternative view predicts worse performance for the change as compared with the continuation groups even in the silent control conditions, and we did not observe such a difference in RTs in any of the experiments [Exp. 1, $t(34)=0.21$; Exp. 2, $t(34)=0.51$; Exp. 3, $t(34)=0.73]$. Therefore, it is unlikely that the novelty of the task in Phase 2 was responsible for the disruptive effect of suppression in the change group. No diminution of the disruption was apparent during the 24 trials of Phase 2 in Experiments 1 and 3. Although Experiment 2 did show a decline over the 24 trials, the disruption remained significant. The effect thus appears to be robust and long-lasting. Furthermore, we have shown that it occurs equally, whether the switch is from a physical to a numerical judgment, or the reverse. We have also shown that the effect is not caused by the need to hold instructions for both judgment tasks in memory simultaneously.

We can conclude, therefore, that suppression has no reliable effect when the mapping of responses is based on a continuation of a recently acquired habit, but disrupts performance in a newly switched condition in which the habit must be overridden. This extends the results of earlier studies, concerned with rapid switching, to our more long-
Table 3 Summary of three-way (Group $\times$ Size Congruity $\times$ Concurrent Task) ANOVAs on RTs in the present experiments and Saeki (2007)

*Significant result. ${ }^{+}$Marginally
significant result.

\begin{tabular}{lllll}
\hline & Saeki (2007) & Experiment 1 & Experiment 2 & Experiment 3 \\
\hline Size judgment in Phase 2 & Numerical & Physical & Physical & Numerical \\
Instructions & Different & Different & Same & Same \\
Group (G) & $p=.85$ & $p=.01^{*}$ & $p=.17$ & $p=.15$ \\
Size congruity (C) & $p<.001^{*}$ & $p<.001^{*}$ & $p<.001^{*}$ & $p<.001^{*}$ \\
Concurrent task (T) & $p=.08^{+}$ & $p<.001^{*}$ & $p<.001^{*}$ & $p<.001^{*}$ \\
$\mathrm{G} \times \mathrm{T}$ & $p=.03^{*}$ & $p<.001^{*}$ & $p<.001^{*}$ & $p=.05^{*}$ \\
$\mathrm{G} \times \mathrm{C}$ & $p=.73$ & $p<.001^{*}$ & $p<.001^{*}$ & $p=.60$ \\
$\mathrm{C} \times \mathrm{T}$ & $p=.08^{+}$ & $p=.83$ & $p<.001^{*}$ & $p<.001^{*}$ \\
$\mathrm{G} \times \mathrm{T} \times \mathrm{C}$ & $p=.60$ & $p=.89$ & $p=.25$ & $p=.02^{*}$ \\
\hline
\end{tabular}


term paradigm. However, unlike the well-established role of phonological coding in tasks involving multiple short-term task switches, the present experiments indicate a disruptive effect of suppression when a single switch is followed by 24 trials requiring the same type of judgment.

To explain our results, comparison of the demands placed on switching in the two different paradigms may be helpful. In rapid task switching, negative effects of suppression have been observed when trial-to-trial uncertainty is high (Saeki \& Saito, 2009). Thus, the problem appears to be how participants keep track of where they are in a sequence of alternating tasks, a process that is probably facilitated by the subvocalization of appropriate cues (e.g., "plus, minus, plus . . ." in the case of switching between addition and subtraction tasks). Our results suggest that the presence of trial-totrial uncertainty is not necessary for the occurrence of a suppression effect. In our long-term switching task, there was no sequential uncertainty. Instead, a recently acquired habit had to be resisted in order to perform the new task judgment efficiently. It seems plausible to assume that a subvocal cue (e.g., "number, number, number, ...") may help strengthen the newly instructed task and inhibit its physical-size competitor. In proposing such an interpretation, it is important to note that even though responding is slowed by articulatory suppression, performance continues to be highly accurate even in the change group. This aspect of the results suggests that although the phonological loop may facilitate speedy access to the task goal, it is not necessary for accurate performance. This is also the case for the rapid-switching paradigm, suggesting in both cases that a verbal code facilitates but is not essential for performance.

Congruity effects and articulatory suppression

A second aspect of our study concerned the effect of articulatory suppression on performance at a more micro, within-trial level, through manipulation of the congruity between physical and numerical size. The first point to note is that we did get very clear congruity effects in all three experiments and in Saeki (2007), confirming that this is very a robust phenomenon. We did, however, find that when the task was to compare physical size (Exps. 1 and 2), the congruity effect was bigger in the change group, whereas the congruity effects were the same for both groups when the task was to compare numerical size (Exp. 3 and Saeki, 2007).

To investigate what might have caused this interaction, we examined what happened to the congruity effect in Blocks 1-3 (i.e., in Phase 1), using data from across the three experiments. In the summary shown in Fig. 5, it is important to appreciate that data have been averaged across all six cycles of the experiment, and therefore Block 1 followed immediately after Block 4 in all except the very first cycle. Figure 5 shows that we saw the same asymmetry in Block 1 that we reported in Block 4 (i.e., Phase 2). Thus, when the task was to compare numerical size, the congruity effect was the same for both the change and continuation groups, whereas when the task was to compare physical size, it was bigger for the change group. However, Fig. 5 also shows that the increase in the congruity effect for physical-size judgments was greatly reduced in Blocks 2 and 3. Overall, we see that the congruity effect for physicalsize judgments was larger following a series of numericalsize judgments than after other physical judgments, whereas the congruity effect for numerical-size judgments showed no corresponding effect. We interpret this time course as suggesting that the change in the congruity effect for physical-size judgments reflects persisting raised activation of the previous, competing numerical-size feature dimension and its corresponding responses, and that this takes some time to dissipate (bearing in mind that each block contained 24 trials). We assume that the reason this pattern was observed for one type of judgment and not for the other reflects an asymmetry between the two congruity effects. A preexisting asymmetry is suggested by evidence that the impact of irrelevant numerical size on physical-size judgments emerges only gradually in child development (Girelli, Lucangeli, \& Butterworth, 2000) and is typically smaller than the impact of irrelevant physical size on numerical-size judgments (e.g., Henik \& Tzelgov 1982; see also Fig. 5 and the Table 4). Accordingly, we suggest that the numerical feature dimension has to be more strongly activated in order to compete with the physical feature dimension for response selection than is true in the converse situation, and that this effect persists over the first few trials of a change to the physical-size task. We do not overlook, however, the fact that a congruity effect was always observed for each type of judgment, indicating that stimulus presentation on each trial always led to some automatic activation of both the physical-size and numerical-size feature dimensions, which delayed responses on incongruent relative to congruent trials.

The question arises, then, as to whether or not the congruity effect is influenced by articulatory suppression. We had no clear predictions on this issue, and did not find a consistent pattern across our series of experiments. Neither Saeki (2007) nor our own Experiment 1 showed a reliable interaction. Interactions were found in both Experiments 2 and 3, but in opposite directions, with Experiment 2 showing an increase in the congruity effect under articulatory suppression, whereas Experiment 3 showed a decreased effect relative to the tapping control. Some researchers have proposed, 
Fig. 5 Mean congruity effects for the numerical-size judgment (left) and the physical-size judgment (right) tasks of Phase 1 (Blocks 1-3) in the change and continuation groups. The data have been averaged across all six cycles of the experiment. The bars represent standard errors

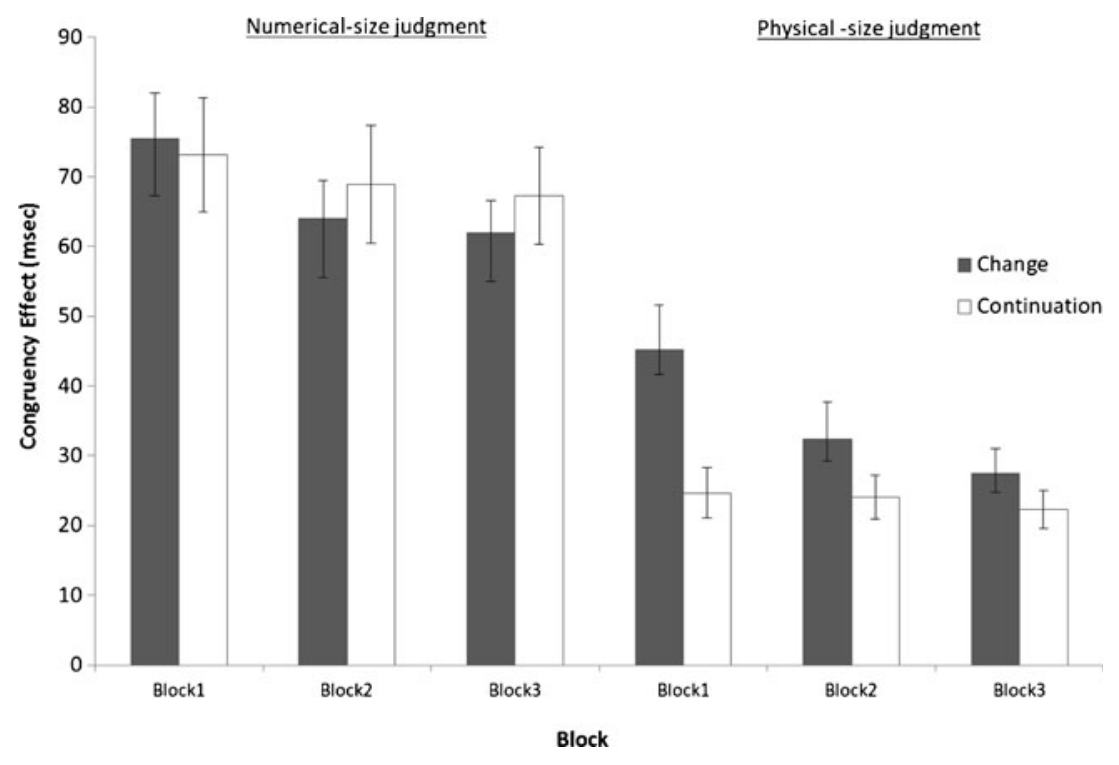

as an explanation of the congruity effect, that numerical size and physical size are initially processed separately and interact at a decision level (Santes \& Verguts, 2011; Schwarz \& Heinze, 1998). It seems likely that the size judgment task includes at least two levels of decision: task decision and response selection. Task decision is widely assumed to precede response selection in task-switching studies (see, e.g., Meiran, Kessler, \& Adi-Japha, 2008; Monsell, 2003; Rubinstein, Meyer, \& Evans, 2001), and a recent study showed that the task-decision process was differentiated from the response-selection process (Braverman \& Meiran, 2010). As was described above, we assume that the phonological loop may support resolving conflict during the task-decision process, not during the response-selection process. The results may indicate that the congruency effect might operate at the level of response selection, uninfluenced by the task-decision process, resulting in the absence of a coherent interaction between congruity and suppression.

The phonological loop and two types of control/operation

In theoretical terms, our findings can be broadly understood in terms of Norman and Shallice's (1986) model of cognitive control, according to which the selection of an action depends on interactions between a limited-capacity "supervisory attentional system" and a set of lower-level "action schemata." Schemata are activated by environmental cues and compete with one another to control behavior. In routine circumstances, the outcome of the competition is determined by automatic processes that operate entirely within the lower level. However, in the present experiment, environmental cues alone were insufficient to trigger the required schema. In such circumstances, input from the higher-level supervisory attentional system is necessary to bias the competition toward the favored schema. The fact that error rates were low in all conditions indicated that executive control was possible, regardless of our concurrent tasks. The fact that articulatory suppression consistently slowed performance indicates a role for subvocal supplementation of the biasing process, a supplementation that appears to have been necessary only when there was competition from a competing habit. The lack of consistency in the effect of our concurrent tasks on the size congruity effect suggests a Stroop-like phenomenon that changes unpredictably from trial to trial and that may operate at a lower level of control.

Such a differentiation between levels of control is captured well by the distinction between procedural and declarative working memory proposed by Oberauer (2009). Procedural working memory concerns the range of implicit operations that underpin working memory, in contrast to those declarative features of which we are typically aware, and that are the focus of the bulk of current working memory research. It could be argued that subvocalization is a process whereby explicit and declarative forms of working memory are used to facilitate the implicit procedural system, providing an ongoing set of cues that protected action from disruption by recent habits in our present study, or that provided helpful serial-ordering cues in earlier studies of rapid switching. If this is the case, one might regard the two task-switching paradigms as providing a means of studying what may prove to be a much more extensive range of situations in which the phonological loop assists in the control of action.

In conclusion, our results suggest that the phonological loop can play an important role in the control of action, even when no sequential uncertainty is present. As such, it links recent research on working memory with the classic studies on the verbal control of action by Luria (1961) and Vygotsky (1962). 


\section{Appendix}

Table 4 Mean reaction times in Phase 1 by trial types across Experiments 1-3, presented separately for the first, second, and third blocks

\begin{tabular}{|c|c|c|c|c|c|c|}
\hline & \multicolumn{2}{|l|}{ First } & \multicolumn{2}{|l|}{ Second } & \multicolumn{2}{|l|}{ Third } \\
\hline & Congruent & Incongruent & Congruent & Incongruent & Congruent & Incongruent \\
\hline \multicolumn{7}{|l|}{ Experiment 1} \\
\hline Continuation (physical size) & $418.20(14.38)$ & $444.03(17.09)$ & $409.14(12.99)$ & $439.19(13.31)$ & $416.48(12.36)$ & $441.92(14.23)$ \\
\hline Change (numerical size) & $452.73(21.11)$ & $542.05(24.80)$ & $444.47(20.61)$ & $527.36(23.89)$ & $452.28(20.48)$ & $521.33(24.45)$ \\
\hline \multicolumn{7}{|l|}{ Experiment 2} \\
\hline Continuation (physical size) & $402.31(10.99)$ & $425.80(12.34)$ & $411.86(11.01)$ & $429.95(12.70)$ & 408.19 (11.99) & $433.59(14.12)$ \\
\hline Change (numerical size) & $491.70(27.98)$ & $553.20(28.17)$ & $496.43(27.90)$ & $551.61(27.29)$ & $488.03(25.36)$ & $543.03(26.12)$ \\
\hline \multicolumn{7}{|l|}{ Experiment 3} \\
\hline Continuation (numerical size) & $481.65(17.85)$ & $554.80(22.60)$ & $489.09(18.84)$ & $557.98(23.97)$ & $485.25(19.92)$ & $552.60(22.33)$ \\
\hline Change (physical size) & $411.52(11.94)$ & $456.77(14.99)$ & $412.28(12.00)$ & $444.73(13.46)$ & $408.99(11.88)$ & $436.44(12.38)$ \\
\hline
\end{tabular}

Standard errors are in parentheses. We conducted the ANOVA using size congruity (congruent and incongruent) and block (1-3) as independent variables. The main effect of size congruity was found on every block in all experiments. We also found significant interactions between congruity and block in the change group of Experiment $1, F(2,34)=3.57, M S E=1,043, p<.05, \eta_{\mathrm{p}}{ }^{2}=.17$, and in the change group of Experiment 3, $F(2,34)$ $=5.06, M S E=1,043, p<.05, \eta_{\mathrm{p}}{ }^{2}=.22$, indicating that the RTs in the second block were shorter than those in the first block only on incongruent trials

\section{References}

Baddeley, A. D. (1996). Exploring the central executive. Quarterly Journal of Experimental Psychology, 49A, 5-28. doi:10.1080/ 027249896392784

Baddeley, A. (2007). Working memory, thought, and action. Oxford: Oxford University Press.

Baddeley, A., Chincotta, D., \& Adlam, A. (2001). Working memory and the control of action: Evidence from task switching. Journal of Experimental Psychology. General, 130, 641-657. doi:10.1037/ 0096-3445.130.4.641

Baddeley, A. D., \& Hitch, G. J. (1974). Working memory. In G. H. Bower (Ed.), The psychology of learning and motivation: Advances in research and theory (Vol. 8, pp. 47-89). New York: Academic Press.

Besner, D., \& Coltheart, M. (1979). Ideographic and alphabetic processing in skilled reading of English. Neuropsychologia, 17, 467472 .

Braverman, A., \& Meiran, N. (2010). Task conflict in task switching. Psychological Research, 74, 568-578.

Bryck, R. L., \& Mayr, U. (2005). On the role of verbalization during task set selection: Switching or serial order control? Memory \& Cognition, 33, 611-623. doi:10.3758/BF03195328

Dehaene, S., \& Akhavein, R. (1995). Attention, automaticity, and levels of representation in number processing. Journal of Experimental Psychology: Learning, Memory, and Cognition, 21, 314326. doi:10.1037/0278-7393.21.2.314

Duncan, J., Parr, A., Woolgar, A., Thompson, R., Bright, P., Cox, S. ... Nimmo-Smith, I. (2008). Goal neglect and Spearman's g: Competing parts of a complex task. Journal of Experimental Psychology: General, 137, 131-148. doi:10.1037/0096-3445.137.1.131

Emerson, M. J., \& Miyake, A. (2003). The role of inner speech in task switching: A dual-task investigation. Journal of Memory and Language, 48, 148-168.
Girelli, L., Lucangeli, D., \& Butterworth, B. (2000). The development of automaticity in accessing number magnitude. Journal of Experimental Child Psychology, 76, 104-122.

Goschke, T. (2000). Intentional reconfiguration and involuntary persistence in task set switching. In S. Monsell \& J. Driver (Eds.), Control of cognitive processes: Attention and performance XVIII (pp. 331-355). Cambridge: MIT Press.

Henik, A., \& Tzelgov, J. (1982). Is three greater than five? The relation between physical and semantic size in comparison tasks. Memory \& Cognition, 10, 389-395. doi:10.3758/BF03202431

Kane, M. J., \& Engle, R. W. (2003). Working-memory capacity and the control of attention: The contributions of goal maintenance, response competition, and task set to Stroop interference. Journal of Experimental Psychology. General, 132, 47-70. doi:10.1037/ 0096-3445.132.1.47

Luria, A. R. (1961). The development of the regulatory role of speech. In J. Tizars (Ed.), The role of speech in the regulation of normal and abnormal behavior (pp. 50-96). New York: Liveright.

Meiran, N., Kessler, Y., \& Adi-Japha, E. (2008). Control by action representation and input selection (CARIS): A theoretical framework for task switching. Psychological Research, 72, 473-500. doi:10.1007/s00426-008-0136-8

Miyake, A., Emerson, M., Padilla, F., \& Ahn, J.-C. (2004). Inner speech as retrieval aid for task goal: The effect of cue type and articulatory suppression in random task cuing paradigm. Acta Psychologica, 115, 123-142.

Miyake, A., \& Shah, P. (1999). Toward unified theories of working memory: Emerging general consensus, unresolved theoretical issues, and future research directions. In A. Miyake \& P. Shah (Eds.), Models of working memory: Mechanisms of active maintenance and executive control (pp. 442-482). Cambridge: Cambridge University Press.

Monsell, S. (1996). Control of mental processes. In V. Bruce (Ed.), Unsolved mysteries of mind: Tutorial essays in cognition (pp. 93148). Hove: Erlbaum. 
Monsell, S. (2003). Task switching. Trends in Cognitive Sciences, 7, 134-140. doi:10.1016/S1364-6613(03)00028-7

Norman, D. A., \& Shallice, T. (1986). Attention to action: Willed and automatic control of behavior. In R. J. Davidson, G. E. Schwartz, \& D. Shapiro (Eds.), Consciousness and self-regulation: Advances in research (Vol. 4, pp. 1-18). New York: Plenum Press.

Oberauer, K. (2007). Activation, binding, and selective access. An embedded three-component framework for working memory. In N. Osaka, R. Logie, \& M. D'Esposito (Eds.), The cognitive neuroscience of working memory (pp. 351-368). Oxford: Oxford University Press.

Oberauer, K. (2009). Design for a working memory. In B. H. Ross (Ed.), The psychology of learning and motivation: Advances in research and theory (Vol. 51, pp. 45-100). San Diego: Elsevier Academic Press. doi:10.1016/S0079-7421(09)51002-X

Rubin, O., \& Meiran, N. (2005). On the origins of the task mixing cost in the cuing task-switching paradigm. Journal of Experimental Psychology: Learning, Memory, and Cognition, 31, 1477-1491. doi:10.1037/0278-7393.31.6.1477

Rubinstein, J. S., Meyer, D. E., \& Evans, J. E. (2001). Executive control of cognitive processes in task switching. Journal of Experimental Psychology. Human Perception and Performance, 27, 763-797.

Saeki, E. (2007). Phonological loop and goal maintenance: Effect of articulatory suppression in number-size consistency task. Psychologia, 50, 122-131. doi:10.2117/psysoc.2007.122

Saeki, E., \& Saito, S. (2004a). Effect of articulatory suppression on task-switching performance: Implications for models of working memory. Memory, 12, 257-271.
Saeki, E., \& Saito, S. (2004b). The role of the phonological loop in task switching performance: The effect of articulatory suppression in the alternating runs paradigm. Psychologia, 47, 35-43.

Saeki, E., \& Saito, S. (2009). Verbal representation in task order control: An examination with transition and task cues in random task switching. Memory \& Cognition, 37, 1040-1050. doi:10.3758/MC.37.7.1040

Saeki, E., Saito, S., \& Kawaguchi, J. (2006). Effect of responsestimulus interval manipulation and articulatory suppression on task switching. Memory, 14, 965-976.

Santes, S., \& Verguts, T. (2011). The size congruity effect: Is bigger always more? Cognition, 118, 94-110.

Schneider, D. W., \& Anderson, J. R. (2010). Asymmetric switch costs as sequential difficulty effects. Quarterly Journal of Experimental Psychology, 63, 1873-1894. doi:10.1080/17470211003624010

Schwarz, W., \& Heinze, H.-J. (1998). On the interaction of numerical and size information in digit comparison: A behavioral and eventrelated potential study. Neuropsychologia, 36, 1167-1179.

Shallice, T. (2002). Fractionation of the supervisory system. In D. T. Stuss \& R. T. Knight (Eds.), Principles of frontal lobe function (pp. 261-277). Oxford: Oxford University Press.

Stuss, D. T., \& Knight, R. T. (2002). Principles of frontal lobe function. Oxford: Oxford University Press.

Tzelgov, J., Meyer, J., \& Henik, A. (1992). Automatic and intentional processing of numerical information. Journal of Experimental Psychology: Learning, Memory, and Cognition, 18, 166-179. doi:10.1037/0278-7393.18.1.166

Vygotsky, L. S. (1962). Thought and language. Cambridge, MA: MIT Press. Original work published 1934. 